J. Phys. IV France 127 (2005) 3-8

(C) EDP Sciences, Les Ulis

DOI: $10.1051 / \mathrm{jp} 4: 2005127001$

\title{
Source X par agrégats : contrôle et optimisation des paramètres gouvernant l'interaction laser de puissance - agrégats de gaz rare
}

\author{
E. Lamour ${ }^{1}$, C. Prigent ${ }^{1}$, J.P. Rozet ${ }^{1}$, D. Vernhet ${ }^{1}$, O. Gobert ${ }^{2}$, P. Meynadier ${ }^{2}$, \\ D. Normand ${ }^{2}$ et M. Perdrix ${ }^{2}$ \\ ${ }^{1}$ GPS-PIIM, Universités Paris 6, Campus Boucicaut, 140 rue de Lourmel, \\ 75015 Paris, France \\ ${ }^{2}$ CEA Saclay, DSM/DRECAM/SPAM, Gif-sur-Yvette, France
}

\begin{abstract}
Résumé. Les expériences que nous avons réalisées sur le Laser Ultra Court Accordable du CEA Saclay permettent d'observer l'émission de photons $\mathrm{X}$ dans la gamme 1-5 keV lors de l'irradiation d'agrégats de gaz rare $\left(\mathrm{Ar}, \mathrm{Kr}\right.$ et $\mathrm{Xe}$ comprenant entre $10^{3}$ et $10^{6}$ atomes par agrégat) avec un laser femtoseconde de puissance $\left(\mathrm{I}_{\text {pic }}\right.$ jusqu'à $10^{17} \mathrm{~W} / \mathrm{cm}^{2}$ ). En plus de la distribution des états de charge des ions responsables de l'émission $\mathrm{X}$, la technique de spectroscopie $\mathrm{X}$ que nous utilisons permet de mesurer les taux absolus de photons émis dans $4 \pi$ par impulsion laser en fonction des paramètres gouvernant l'interaction dans des conditions contrôlées. Nous avons déterminé la sensibilité des paramètres physiques régissant la production du rayonnement $\mathrm{X}$ pendant l'interaction, ce qui permet d'accéder à l'optimisation de cette source. Cet article est plus particulièrement dédié aux résultas relatifs à l'évolution du taux d'X avec l'éclairement laser, d'une part, et avec la durée de l'impulsion laser, d'autre part.
\end{abstract}

\section{INTRODUCTION}

L'étude de l'interaction d'impulsions laser ultracourtes de haute intensité avec la matière (de l'atome au solide) a reçu une attention toute particulière ces dernières années depuis l'avènement de la technique de la CPA (Chirp Pulse Amplification). Plus spécifiquement, dans le cas d'agrégats soumis à des champs électriques très intenses $\left(\mathrm{F}>10^{9} \mathrm{~V} / \mathrm{cm}\right)$, le couplage énergétique entre la lumière laser et la matière est très efficace. Cette efficacité se traduit par toute une série d'observations expérimentales très différentes de ce qui se passe avec des atomes, des petites molécules voire des solides: production d'ions très chargés ayant des énergies jusqu'au MeV [1], d'électrons "chauds" de quelques keV [2] et de photons X dans le domaine du $\mathrm{keV}[3,4,5,6]$. Les agrégats forment une cible unique réunissant les avantages des cibles gazeuses et solides. En premier lieu, une cible d'agrégats sous l'influence d'une lumière laser intense ne produit pas de débris pouvant affecter l'optique associée à l'expérience. Ensuite, les agrégats communément utilisés possèdent une densité locale d'atomes proche de celle des solides $\left(\sim 10^{22} \mathrm{at} / \mathrm{cm}^{3}\right)$ tout en ayant une taille (quelques dizaine de nanomètres) bien inférieure à la longueur d'onde du laser (400-800 nm). En première approximation, le champ électrique peut donc être considéré comme étant le même sur chaque atome cible (épaisseur de peau $\sim$ taille de l'agrégat) contrairement à une cible solide où des effets d'écrantage du champ laser apparaissent en profondeur. Différents modèles ont été développés [7, 8, 9] pour expliquer les mécanismes d'absorption de l'énergie laser, la dissipation de cette énergie au sein de l'agrégat ainsi que le mode d'expansion de l'agrégat. Très récemment, de nouvelles expériences principalement dédiées à l'étude de l'émission des ions et/ou des électrons $[10,11]$ remettent en cause certains points des modèles comme, par exemple, l'observation très controversée [10] d'un pic d'électrons chauds prédit par le modèle nanoplasma standard unidimensionnel [7].

Les expériences que nous avons réalisées sur le Laser Ultra Court Accordable du CEA Saclay permettent d'observer l'émission de photons $\mathrm{X}$ dans la gamme 1-5keV lors de l'irradiation d'agrégats de gaz rare ( $\mathrm{Ar}, \mathrm{Kr}$ et Xe comprenant entre $10^{3}$ et $10^{6}$ atomes par agrégat) avec un laser femtoseconde 
de puissance $\left(\mathrm{I}_{\text {pic }}\right.$ jusqu'à $\left.10^{17} \mathrm{~W} / \mathrm{cm}^{2}\right)$. Ce rayonnement $\mathrm{X}$, dont l'absorption est négligeable à travers l'agrégat, provient de la production, au cours de l'interaction, d'ions fortement multichargés présentant des lacunes en couche interne. Grâce à la technique de spectroscopie X développée par l'équipe, nous avons observé, dans le cas d'agrégats d'argon, des ions $\mathrm{Ar}^{\mathrm{q}+}$ avec $12 \leq \mathrm{q} \leq 16$ ayant une lacune en couche $\mathrm{K}[4,5]$ et, dans le cas d'agrégats de xénon, des ions $\mathrm{Xe}^{\mathrm{q}+}$ avec $24 \leq \mathrm{q} \leq 34$ ayant des lacunes en couche L [6]. Le rayonnement X constitue une bonne observable de la dynamique de l'interaction puisque la désexcitation des états excités peuplés a lieu sur des temps très courts, de quelques 10 à 100 fs. Elle permet donc d'avoir des informations sur les phénomènes d'interaction bien avant d'éventuelles recombinaisons. Ceci est cohérent avec le fait que les espèces ioniques observées par spectroscopie $\mathrm{X}$ sont beaucoup plus multichargés (par exemple jusqu'à l' $\mathrm{Ar}^{16+}$ ) que celles observées en spectroscopie d'ions (au plus $\mathrm{Ar}^{10+}$ [12]). Outre la distribution des états de charge des ions responsables de l'émission $X$, notre technique permet également de mesurer les taux absolus de photons émis dans $4 \pi$ par impulsion laser. Par exemple, dans des conditions optimales, jusqu'à $10^{9}$ photons d'environ $4,4 \mathrm{keV}$ ont été mesurés avec des agrégats de xénon. En dehors des applications potentielles (expériences XPS, voire XPS résolues en temps par exemple), le but de nos études est de comprendre la dynamique des processus mis en jeu lors de l'interaction. En particulier, il est nécessaire de savoir comment des électrons, initialement produits par ionisation par effet de champ, atteignent une énergie suffisamment élevée pour conduire, via des collisions électrons - ions, à la création de lacunes en couche interne pour les ions multichargés produits. Puisque les mécanismes impliqués dans le mode de chauffage des électrons sont encore très controversés, nous avons effectué des mesures quantitatives précises en fonction des paramètres physiques gouvernant l'interaction tels que l'intensité, la polarisation, la durée de l'impulsion et la longueur d'onde du laser, la nature, la taille et la densité des agrégats. L'ensemble des données expérimentales acquises nous permet de déterminer la sensibilité des paramètres physiques régissant la production du rayonnement $\mathrm{X}$ pendant l'interaction et ainsi d'accéder à l'optimisation de cette source. Par ce biais, on obtient aussi des indications sur la dynamique de l'interaction ainsi que sur les processus mis en jeu.

Nos premiers résultats concernaient la mise en évidence de lois d'échelle avec l'intensité laser et avec la pression [3, 4] ainsi que l'existence d'un seuil en intensité pour la production des photons X [5]. Avec les agrégats de xénon, nous avons montré que l'émission X ne dépend que faiblement de la longueur d'onde du laser [6] (comparaison IR-800nm/UV-400 nm) ce qui remet en cause d'autres études expérimentales [13]. Pour ces études, des divergences marquées apparaissent aussi au niveau de l'interprétation des spectres X. Dans l'article présenté ici, nous reviendrons, dans la section 3, sur l'évolution des photons $\mathrm{X}$ avec l'éclairement et plus particulièrement sur les conséquences de nos observations. Dans la section 4, de nouveaux résultats sur l'évolution de l'émission $\mathrm{X}$ avec la durée de l'impulsion laser seront discutés.

\section{EXPÉRIENCE}

Les différentes campagnes d'expériences que nous avons réalisées ont été effectuées auprès du serveur LUCA du SPAM/DRECAM au CEA/Saclay. Cette source femtoseconde est constituée d'une chaîne laser à Saphir dopé au Titane délivrant, à un taux de répétition de $20 \mathrm{~Hz}$, des impulsions IR $(\lambda=800 \mathrm{~nm})$, d'énergie modulable entre 0 et $100 \mathrm{~mJ}$ et de durée d'impulsion comprise entre 50 et $2000 \mathrm{fs}$. A l'aide d'une lentille de distance focale $f=480 \mathrm{~mm}$, nous avons pu ainsi faire varier l'éclairement laser de $10^{14}$ à $10^{17} \mathrm{~W} / \mathrm{cm}^{2}$ au foyer du faisceau laser. Le jet d'agrégats est, quant à lui, produit par expansion adiabatique à travers une buse conique d'un gaz rare sous pression. Cette pression amont $\mathrm{P}_{0}$ contrôle la taille de nos agrégats suivant des lois d'échelles (taille $\propto \mathrm{P}_{0}^{2}$ ) établies par Hagena [14]. Il est à noter que de nombreuses études confirmant ces évolutions permettent aussi de déterminer le taux d'agrégation [15] et donc le nombre d'agrégats contenus dans la zone d'interaction. En ce qui concerne le rayonnement X, le diagnostic s'effectue à l'aide d'un système de détection calibré en absolu et composé de 2 détecteurs $\mathrm{Si}(\mathrm{Li})$ (spectroscopie dite de "basse résolution" : de l'ordre de $150 \mathrm{eV}$ à $3 \mathrm{keV}$ ) et d'un spectromètre à 
cristal de Bragg de haute résolution (de l'ordre de 1,5 eV à 3 keV) et de grande transmission. A l'aide de ce dernier, nous avons réalisé une spectroscopie fine du rayonnement $\mathrm{X}$ donnant notamment accès aux transitions suivantes:

i) dans le cas des agrégats d'Ar, $2 p \rightarrow 1 s$ issues des ions d'états de charge allant de l'Ar' ${ }^{12+}$ jusqu'à $l^{\prime} \mathrm{Ar}^{16+}[4,5]$ et $3 p \rightarrow 1 s$ issues des ions $\mathrm{Ar}^{16+}$ et $\mathrm{Ar}^{15+}$,

ii) dans le cas des agrégats de $\mathrm{Xe}, 3 d_{3 / 2} \rightarrow 2 p_{1 / 2}$ et $3 d_{5 / 2} \rightarrow 2 p_{3 / 2}$ issues des ions $\mathrm{Xe}^{q+}$ avec $q \geq 24[6]$.

Enfin la connaissance de la transmission, de l'angle solide et de l'efficacité de l'ensemble du système de détection permet de mesurer des taux absolus de production du rayonnement $\mathrm{X}$. Notre technique nous a permis de suivre l'évolution de ces taux en fonction d'un, et d'un seul, paramètre à la fois ; c'est-à-dire en contrôlant au mieux l'ensemble de tous les autres paramètres susceptibles d'influer sur l'intensité de la source $\mathrm{X}$.

\section{3. ÉVOLUTION DU TAUX D’X AVEC L'ÉCLAIREMENT LASER}

Nous avons étudié l'évolution du taux de photons X produits en fonction de l'éclairement laser (I) pour des agrégats d'Ar et de $\mathrm{Kr}$ avec comme longueur d'onde laser $800 \mathrm{~nm}$ et durée d'impulsion 55 fs [4, 5]. Avec des agrégats de xénon dans les mêmes conditions laser, on retrouve le même comportement (figure 1) que celui déjà observé pour les autres agrégats de gaz rare [5]:

i) augmentation rapide du taux de photons au-dessus d'un éclairement seuil $\mathrm{I}_{\text {seuil }} \sim 1,510^{15} \mathrm{~W} / \mathrm{cm}^{2}$,

ii) puis, évolution avec l'éclairement en $\mathrm{I}^{3 / 2}$ au-dessus de $\mathrm{I} \sim 410^{15} \mathrm{~W} / \mathrm{cm}^{2}$ (cette évolution est appelée régime de saturation).

Ce comportement global est similaire à celui observé en interaction laser-atome [16]: le signal physique observé augmente uniquement avec le nombre de partenaires contenus dans le volume focal effectif à l'intérieur duquel une intensité seuil est dépassée. Dans le cas de l'interaction laser-agrégat, le nombre de photons $\mathrm{X}$ est également proportionnel au volume focal effectif dès qu'une intensité seuil est atteinte, comme le prévoit d'ailleurs le modèle collisionnel dynamique développé par l'équipe [4, 5]. Cependant, contrairement aux prévisions de ce modèle, l'intensité seuil observée est beaucoup plus petite (un ordre de grandeur plus basse). Expérimentalement on trouve $I_{\text {seuil }} \sim 1-210^{15} \mathrm{~W} / \mathrm{cm}^{2}$ quelle que soit la nature des agrégats pour une durée d'impulsion laser de 50-60 fs. Le fait que le nombre de photons X augmente comme le volume focal effectif a une conséquence directe sur la probabilité d'ionisation en couche interne, qui n'est rien d'autre que le rapport du taux absolu de photons X émis sur le nombre d'atomes émetteurs. Or ce nombre d'atomes émetteurs est lui aussi proportionnel au volume focal effectif (courbe en trait plein de la figure 1). Finalement, dès que l'éclairement laser est supérieur à $\mathrm{I}_{\text {seuil }}$, la probabilité d'ionisation en couche interne est constante. Dans l'exemple présenté figure 1, on obtient une probabilité de l'ordre de quelques $10^{-5}$ par atome. Ces observations soulèvent des questions fondamentales quant aux mécanismes responsables de la création de ces lacunes. A l'heure actuelle, l'énergie moyenne (ou la température) électronique prédite par les modèles n'excède pas une centaine d'eV, quelle que soit l'approche théorique utilisée, ce qui ne peut en aucun cas expliciter l'ionisation en couche interne.

Au-delà de ces questions, on en déduit que pour optimiser la production de photons X, il faut finalement optimiser le volume focal effectif. Sur la figure 2, est représentée l'évolution du volume focal effectif en fonction du diamètre au foyer $\left(\mathrm{w}_{0}\right)$ pour trois énergies laser différentes et une intensité seuil de $1,510^{15} \mathrm{~W} / \mathrm{cm}^{2}$. On remarque immédiatement que les courbes présentent des maxima pour des valeurs "grandes" du diamètre au foyer (par exemple, pour $10 \mathrm{~mJ}$ d'énergie laser, $\mathrm{V}_{\text {foc }}$ est maximal pour $\left.\mathrm{w}_{0}=60 \mu \mathrm{m}\right)$. La conclusion est donc que pour maximiser le taux de photons $\mathrm{X}$ à énergie par impulsion constante, il est préférable d'avoir un "grand" diamètre au foyer correspondant à un "faible" éclairement laser plutôt qu'un "petit" diamètre au foyer correspondant à un "grand" éclairement, pourvu qu'on est atteint l'intensité seuil. 


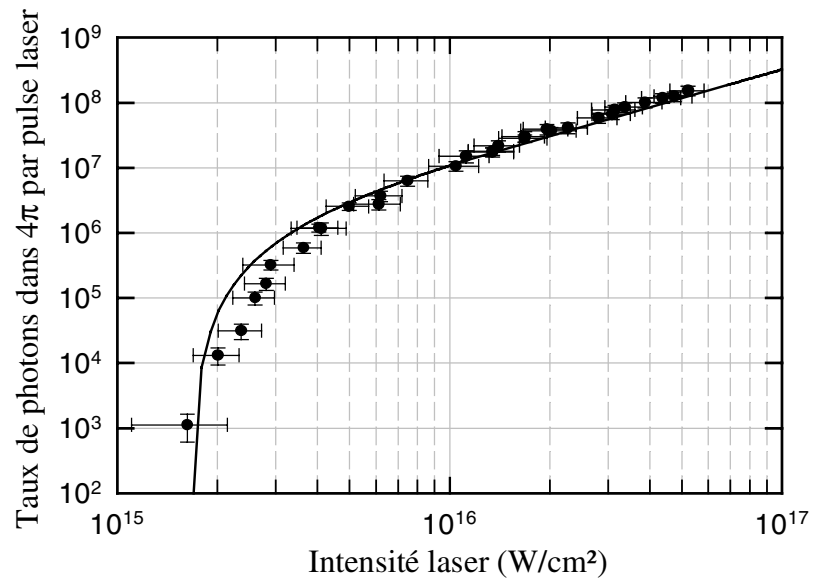

Figure 1. Évolution du taux de photons $X$ d'énergie moyenne de $4,4 \mathrm{keV}$ en fonction de l'éclairement laser (en IR et pour une durée d'impulsion de $60 \mathrm{fs})$ dans le cas d'agrégats de xénon $\left(\mathrm{P}_{0}=8.6\right.$ bar). La courbe en trait plein correspond à l'évolution du volume focal effectif obtenu pour un seuil de $1,5.10^{15} \mathrm{~W} / \mathrm{cm}^{2}$.

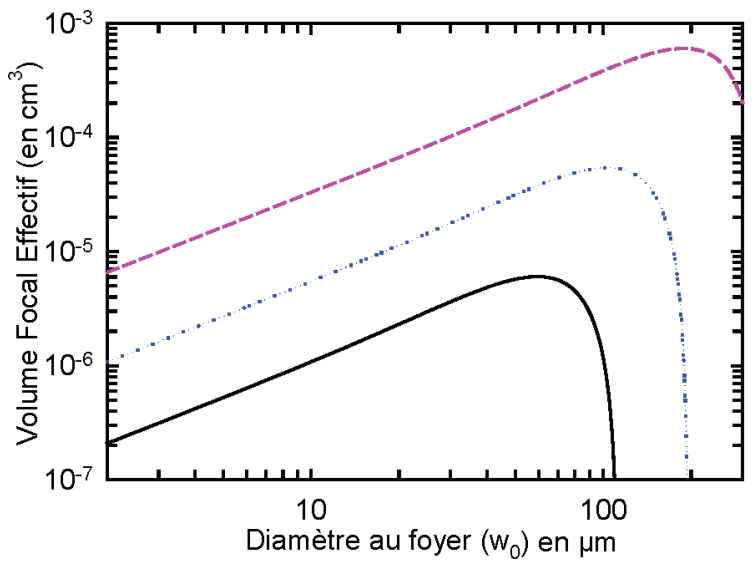

Figure 2. Évolution du volume focal effectif en fonction du diamètre au foyer pour 3 énergies par impulsion laser différentes: $10 \mathrm{~mJ}$ (trait plein), $30 \mathrm{~mJ}$ (en pointillés) et $100 \mathrm{~mJ}$ (en tirets).

\section{4. ÉVOLUTION DU TAUX D’X AVEC LA DURÉE DE L'IMPULSION LASER}

Récemment, nous avons entrepris une étude du taux de photons $X\left(\mathrm{~N}_{\mathrm{X}}\right)$ en fonction de la durée de l'impulsion $(\tau)$ à énergie laser constante (deux exemples sont donnés figures $3 \mathrm{a}$ et $3 \mathrm{~b}$ ) afin de mieux cerner la compétition temporelle des processus de chauffage (Bremsstrahlung inverse, accélération des électrons dans le champ laser...). Il est à noter que ces études ont été effectuées dans le régime de saturation (cf. section 3).

Aussi bien avec des agrégats d'Ar que de Xe, on observe une augmentation brusque du taux de photons jusqu'à un optimum, puis une décroissance lente. Cette décroissance, qui varie en $\tau^{-1 / 2}$, peut facilement s'expliquer par le modèle collisionnel dynamique. Pour cela, rappelons que l'on peut écrire [4, 5]:

$$
\mathrm{N}_{\mathrm{X}} \propto \mathrm{n}_{\text {cycle }} \times \mathrm{V}_{\text {foc eff }}
$$



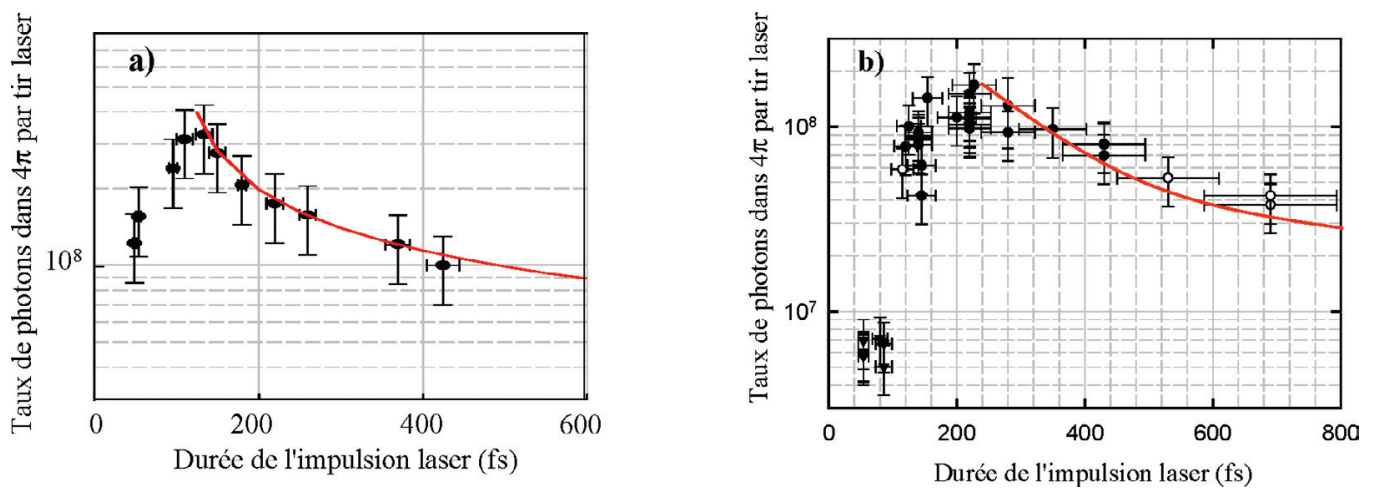

Figure 3. Évolution du taux de photons $\mathrm{X}$ en fonction de la durée de l'impulsion laser (en IR) :

a) dans le cas d'agrégats d'argon $\left(\mathrm{P}_{0}=20\right.$ bar) et pour une énergie par impulsion maintenue égale à $20 \mathrm{~mJ}$,

b) dans le cas d'agrégats de xénon $\left(\mathrm{P}_{0}=3.5\right.$ bar) et pour une énergie par impulsion maintenue égale à $35 \mathrm{~mJ}$. La courbe en trait plein correspond à une évolution du taux de photons en $\tau^{-1 / 2}$, avec $\tau$ la durée de l'impulsion.

avec $\mathrm{n}_{\text {cycle }}$ le nombre de cycles optiques. Or, le volume focal effectif est proportionnel à $\mathrm{I}^{3 / 2}$ (dans le régime de saturation) donc inversement proportionnel à $\tau^{3 / 2}$ et le nombre de cycles optiques est, quant à lui, proportionnel à $\tau$, ce qui conduit bien à une variation du nombre de photons en $\tau^{-1 / 2}$. De plus figure 3, on observe que la position des optima dépend, quant à elle, de la nature des agrégats: on trouve un optimum autour de $130 \mathrm{fs}$ pour des agrégats d'Ar (figure 3a) et autour de $210 \mathrm{fs}$ pour des agrégats de Xe (figure $3 \mathrm{~b}$ ) avec une augmentation dans la production des $\mathrm{X}$ plus marquée dans le cas du Xe que pour l'Ar. On peut penser que cela traduit le fait que la compétition temporelle des processus mis en jeu dans la création des lacunes en couche interne est influencée par le type de lacunes crées (lacunes $\mathrm{K}$ pour l'Ar et L pour le Xe). D'autres résultats pour des tailles d'agrégats différentes sont en cours d'analyse. L'observation d'un maximum dans la production des photons X est caractéristique d'un temps de chauffage optimum que doivent avoir les électrons pour ioniser les ions du plasma en couche interne.

\section{CONCLUSION}

Les expériences que nous avons réalisées nous ont permis d'obtenir des mesures quantitatives absolues du rayonnement $\mathrm{X}$ en fonction des paramètres gouvernant l'interaction dans des conditions contrôlées. Ce court papier est dédié aux résultats relatifs à l'évolution du taux d'X avec l'éclairement laser, d'une part, et avec la durée de l'impulsion laser, d'autre part.

Ces mesures mettent en évidence un seuil de production du rayonnement $X$ en intensité laser très bas, du moins beaucoup plus bas que ce que l'on peut prédire actuellement théoriquement [5]. Nous avons aussi déduit que la probabilité d'ionisation en couche interne est constante au-delà de l'éclairement seuil. Enfin, l'étude de l'influence de la durée de l'impulsion laser sur la production des photons $\mathrm{X}$ révèle un temps de chauffage optimal des électrons. Ces résultats doivent donner des indications sur les processus de chauffage et sur la dynamique de l'interaction. Pour optimiser cette source X, nous avons aussi montré, contre toutes idées reçues, qu'il était préférable de travailler avec un "grand" volume effectif et une durée d'impulsion laser pas trop "courte".

Très récemment, de nouvelles mesures quantitatives de l'évolution du taux de photons $X$ avec l'éclairement pour des durées d'impulsion supérieures à 60 fs ainsi que pour une longueur d'onde laser différente ( $400 \mathrm{~nm}$ ) ont été effectuées. Les résultats préliminaires confirment le comportement du taux de photons $\mathrm{X}$ avec le volume focal effectif mais des seuils en intensité encore plus faibles ont été observés. Ces résultats feront l'objet d'une prochaine publication. 


\section{Bibliographie}

[1] Ditmire T., Tisch J.W., Springate E. et al., Nature 386 (1997) 54-56.

[2] Shao Y.L., Ditmire T., Tisch J.W. et al., Phys. Rev. Lett. 77 (1996) 3343-3346.

[3] Dobosz S., Lezius M., Schmidt M. et al., Phys. Rev. A 56 (1997) R2526-R2529.

[4] Rozet J.P., Cornille M., Dobosz S. et al., Physica Scripta T92 (2001) 113-118.

[5] Lamour E., Dreuil S., Gauthier J.C. et al., Proc. of SPIE, Eds G.A.Kyrala and J.C. Gauthier 4506 (2002) 97-105.

[6] Adoui L., Gobert O., Indelicato P. et al., NIMB 205 (2003) 341-345.

[7] Ditmire T., Donnelly T., Rubenchik A.M. et al., Phys. Rev. A 53 (1996) 3379-3402.

[8] Rose Petruck C., Schafer K.J., Wilson K.R. et al., Phys. Rev. A 55 (1997) 1182-1190.

[9] Boyer K., Thompson B.D., McPherson A. et al., J. Phys. B 27 (1994) 4373-4389.

[10] Springate E., Aseyev S.A., Zamith S. et al., Phys. Rev. A 68 (2003) 053201.

[11] Krishnamurthy M., Mathur D. et Kumarappan V., Phys. Rev. A 69 (2004) 033202.

[12] Lezius M., Dobosz S., Normand N. et al., Phys. Rev. Lett. 80 (1998) 261-264.

[13] Borisov A.B., Song X., Frigeni F. et al., J. Phys. B 35 (2002) L461-L468.

[14] Hagena O.F. et Obert W., J. Chem. Phys. 56 (1972) 1793-1799.

[15] Blasco F., Caillaud T. Dorchies F. et al., NIM B 205 (2003) 324-328.

[16] Augst S., Meyerhofer D.D., Strickland D. et al., Phys. Rev. Lett. 63 (1989) 2212-2215. 